\title{
A Preliminary Investigation into the use of Machine Vision Techniques for Automating Facial Paralysis Rehabilitation Therapy
}

\author{
Jane Reilly Delannoy* and Tomás E. Ward ${ }^{* *}$ \\ *Computer Vision and Imaging Laboratory, \\ **Biomedical Engineering Research Group, \\ Department of Computer Science, \\ National University of Ireland, Maynooth \\ Department of Electronic Engineering, \\ National University of Ireland, Maynooth \\ Email:jreilly@cs.nuim.ie \\ Email:tomas.ward@nuim.ie
}

\begin{abstract}
An impaired facial motor system is a common deficit associated with injury to the nervous system such as occurs during stroke or head trauma. Despite the impact Facial Motor System (FMS) damage has on psychological and social aspects of an individual's quality of life, facial motor rehabilitation has received little attention until comparatively recently. In this paper preliminary results of our investigation into the use of machine vision methods for the development of an automatic feedback system are presented. We show by way of experimental results that our system provides initial steps towards the development of a system for enhancing the rehabilitation prospects of individuals suffering from FMS damage. The proposed system will act as an intelligent mirror, providing basic feedback by tracking key facial features during the attempted gesture. Such a system could potentially emulate the therapist, automatically assessing the patient according to standard facial disability measures, allowing a comprehensive record of patient engagement, performance, efficacy and outcome to be constructed in a relatively inexpensive manner.
\end{abstract} processing

Keywords - Neurorehabilitation, facial rehabilitation, active appearance models, image

I INTRODUCTION

Neurorehabilitation is a branch of applied neuroscience, the purpose of which is to aid patient recovery following damage to the nervous system resulting from disease or injury. Such intervention has traditionally focussed on the parts of the nervous system which yield functional improvement in Activities of Daily Living (ADL) such as self-care and independent living. Given the limited rehabilitation resources available, little is done to rehabilitate the aspects of the damaged nervous system which do not directly impact on ADL.

One such aspect is the Facial Motor System (FMS), the dysfunction of which is commonly associated with conditions such as stroke and head injury. In general, most individuals with FMS damage do not receive specific rehabilitation therapy and improvement in the FMS either emerges spontaneously as the system recovers naturally or not at all.

As facial expressions play an important role in everyday communication and conversation, individuals with paralysed expression and facial disfigurement suffer socially and psychologically as well as obviously physically [1]. The recognition that untreated disorders of the FMS has such profound impact on the quality of life of sufferers (and consequently an indirect but powerful effect on ADL) is leading to a new emerging science of facial rehabilitation [2]. While the area is currently in its infancy, already significant research has been conducted into evaluating the relative effectiveness of a range of approaches. Increasingly biofeedback methods (overwhelmingly vision based such as the use of a simple mirror) when used in conjunction with a daily training regime are seen as having a significant role to play in the design of suitable rehabilitation therapy. Such therapy is most effectively and efficiently administered at home and therefore increasingly the onus is on the patient to engage and drive their own recovery.

In this paper we describe a preliminary study in which we investigate the use of machine vision methods to enhance FMS rehabilitation with a view to developing a home-based rehabilitation system. Such a system can act as antelligent mirror which as well as providing basic feedback can track key facial features during attempted gesture. The tracking of these features may allow such a system to emulate the therapist by automatically assessing the patient 
according to standard facial disability measures. This would allow for a more comprehensive record of patient engagement, performance, efficacy and outcome to be constructed in a relatively inexpensive manner. It is this aspect of the intelligent mirror which we explore and develop in this paper.

The rest of this paper is organised as follows. Section II presents an overview of FMS rehabilitation therapy including where appropriate, ICT-driven approaches. Section III is the primary contribution of this paper and describes a pilot study demonstrating how a particular machine vision method called Active Appearance Models (AAM) [3] can be used as part of a system to replicate a standard facial disability scoring procedure which would normally be undertaken by a therapist. Section IV discusses the implications of the results of the experiment before conclusions are drawn in the final section.

\section{FACIAL REHABILITATION APPROACHES}

Facial rehabilitation falls under the specialist physical therapy rehabilitation category. Currently at the practise level it is very dependent on therapist patient interaction and only simple technologies have been used. At a research level the area is attracting increasing interest and a number of machine visionbased research efforts have emerged in recent years [4].

\section{a) Traditional physical therapy approaches}

Traditional approaches have ranged from basic facial muscle manipulation through massage through to electrical stimulation methods in which specific patterns of facial activation are generated by applying electrical current via electrodes positioned at various points on the face. These are old methods and have long since been shown to be relatively ineffective [5].

Currently facial re-education methods are in favour. Facial neuromuscular re-education is a process during which movement is restored through specific and accurate feedback. Appropriate functional patterns of movement are targeted but just as importantly abnormal movement and activity (synkinesis) are targeted for suppression. In some cases biofeedback of the electrical activity of the specific muscles to be targeted is used, while simpler approaches involve the careful use of a mirror for visual feedback. In all cases the therapist is required both for interaction at least initially and subsequently for scoring of performance. Scoring as is common in rehabilitation requires the application of carefully constructed scales.

\section{b) Facial grading scales}

One of the more common facial expressions is that of smiling. The formation of a smile is highly complex, requiring the coordination of all of the major facial muscles groups such as the levator anguli oris, levator labii superioris, orbicularis oculi, risorius and the zygomaticus. As such measuring ones' ability to smile is an ideal test of the overall facial motor system.

Scales are often used to measure the efficacy of treatment and progress towards a functional goal. Common scales are the Sunnybrook, the Sydney and the Peitersen grading systems [6]. More complex grading systems also exist such as the HouseBrackman Facial nerve grading system [7]. Details of this scale with respect to the mouth and the ability to smile are provided in Table 1.

\begin{tabular}{|l|l|l|}
\hline Grade & Description & Characteristics \\
\hline I & Normal & normal function \\
\hline II & Slight & $\begin{array}{l}\text { slight asymmetry in the } \\
\text { mouth }\end{array}$ \\
\hline III & Moderate & $\begin{array}{l}\text { slight weakness with } \\
\text { maximum effort }\end{array}$ \\
\hline IV & $\begin{array}{l}\text { Moderately } \\
\text { Severe }\end{array}$ & $\begin{array}{l}\text { asymmetrical with } \\
\text { maximum effort }\end{array}$ \\
\hline V & Severe & $\begin{array}{l}\text { slight movement of } \\
\text { mouth only }\end{array}$ \\
\hline VI & Total & \multicolumn{2}{|l|}{ no facial function } \\
\hline \multicolumn{2}{|l|}{ Table 1: Extract relating to the mouth and smiling ability }
\end{tabular}

from House-Brackman Facial Nerve Grading System [7]

A variant of the House-Brackman grading system is the House-Brackman score. The HouseBrackman score, illustrated in Figure 1, is based on two measurements; the upwards movement of the brows, and the lateral movement of the corners of the mouth. 1 point is awarded per $0.25 \mathrm{~cm}$ of movement with a total of 8 points. This is currently the most thorough unobtrusive mechanism for measuring FMS, and we use it as the basis for our automated FMS measurement system.
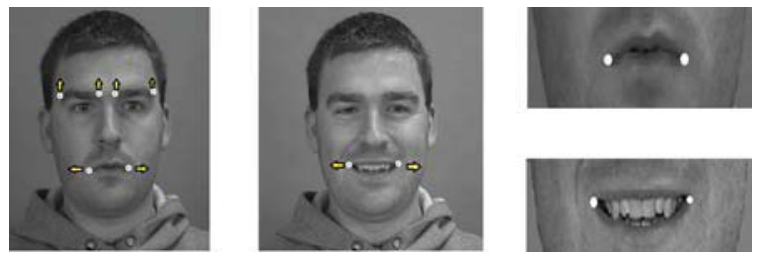

Figure 1: (left) facial aspects measured by HouseBrackman scoring system, (centre) elements of HouseBrackman system concerned with smiling, (right) depicts the points which need to be detected and tracked to score smiling function.

\section{c) Facial image processing approaches}

The first stage in any computer vision based system for measuring facial motor function is one of feature extraction. This involves the process through which information relating to the features of interest are detected and extracted from facial images. AAMs are an example of one such technique which is commonly used for feature detection [3]. In our experimentation we implement AAMs to extract information relating to the shape and location of the 
facial features which are required for HouseBrackman scoring as shown in Figure 1.

\section{PRELIMINARY INVESTIGATIONS}

In this paper we propose a computer vision based system for automatically measuring a patient's ability to perform a smile. There are a number of stages to this procedure, including facial feature detection, image synthesizing, and finally measurement of facial expression symmetry.

\section{a) Active Appearance Models (AAMs)}

Within the realm of facial expression analysis, Active Appearance Models (AAMs) are commonly used for two tasks, firstly feature detection and secondly facial expression synthesis [3]. Effectively AAMs can be viewed as statistical models of the shape and grey level appearance of the face which deform iteratively to fit to new images. The process of matching a new image to an AAM involves finding model parameters which minimise the difference between an image and its synthesised reconstruction image, shown in Figure 2.

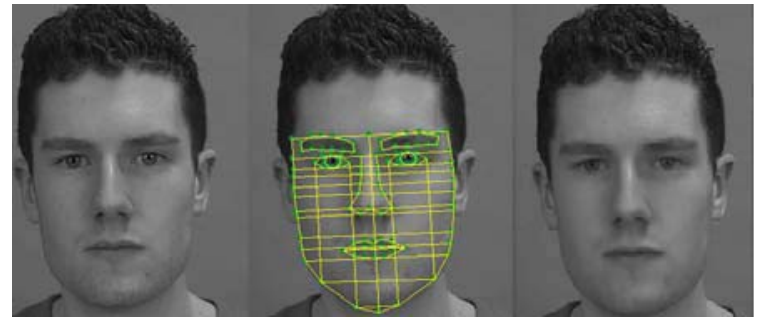

Figure 2: Feature localisation using AAMs, original image (left) image fitted with AAM - shape model (centre),

AAM - synthesized reconstruction image (right)

b) Synthesizing Appropriate Facial Data Sets

Due to the lack of publicly available datasets containing images of individuals experiencing various levels of FMS damage, the first task in our experimentation was to synthesize asymmetrical facial expressions. This was done by combining the left and right sides of two images of the same person - one displaying a neutral expression, and the other displaying a smile expression. Examples of these images are shown in Figure 3. The level of FMS damage shown in these images roughly equates to a House-Brackman scoring of VI (no facial function), on the damaged side of their face.

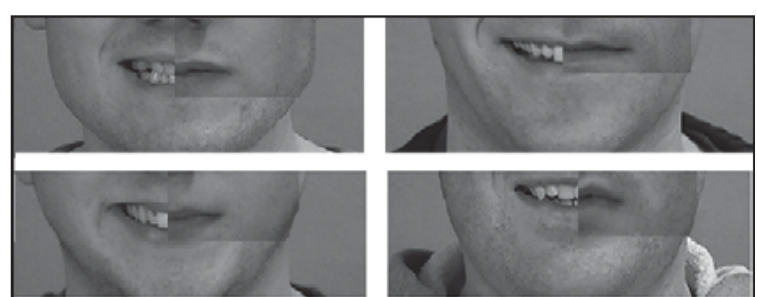

Figure 3: Example of synthesized asymmetrical smiles
Given the images from Figure 3 as input, along with the original smile expression, individual AAMs were trained for each subject. By interpolating between the asymmetrical expression and the smile expression we created a set of synthesised reconstruction images such as those shown in Figure 4 for each subject. These images display similar characteristics as those of patients experiencing various levels of FMS damage and as such provide a valid benchmark to measure the accuracy of our automated FMS measurement system.

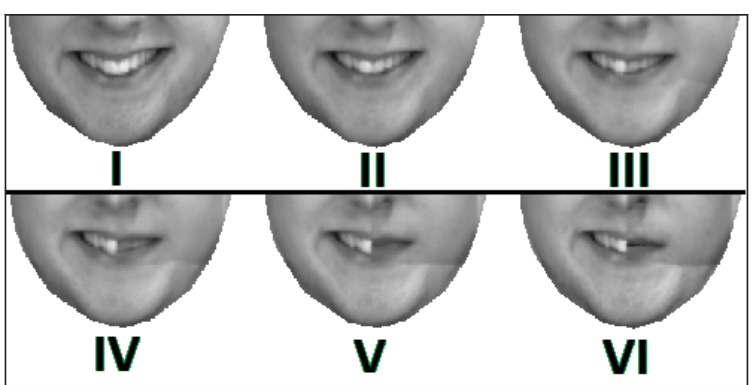

Figure 4: Example of a set of synthesized reconstructed asymmetrical smiles and their equivalent House Brackman scoring

c) Measuring a patient's ability to perform a symmetrical smile.

Our implementation of the House-Brackman scoring system with respect to an individuals' ability to perform a symmetrical smile, is based on measuring the lateral movement of the outer corners of the mouth. For example, using image VI from Figure 4 as a starting point, we measure the distance between the corners of the mouth on this image to those on each of the subsequent images.

This value is interpreted using elements of the House-Brackman scoring system which specifically relate to the lower face -i.e. the lateral movement of the corners of the mouth, as indicated in Table 2 below.

\begin{tabular}{|l|l|l|}
\hline Grade & Description & \multicolumn{1}{c|}{ measurement } \\
\hline I & Normal & $\boldsymbol{d}>1.0$ \\
\hline II & Slight & $1.0<\boldsymbol{d}>0.75$ \\
\hline III & Moderate & $0.5<\boldsymbol{d}>0.75$ \\
\hline IV & $\begin{array}{l}\text { Moderately } \\
\text { Severe }\end{array}$ & $0.25<\boldsymbol{d}>0.5$ \\
\hline V & Severe & $\boldsymbol{d}>0.25$ \\
\hline VI & Total & $\boldsymbol{d}=0$ \\
\hline
\end{tabular}

Table 2: Modified House Brackman scale which we implement. $d=$ distance between location of point on neutral image and that in the test image.

\section{d) Experimental Results}

Our preliminary test set contained frontal images of neutral and smile expressions from 5 healthy subjects. For each subject appropriate levels of FMS damage was synthesised using AAMs. Prior 
to experimentation, these synthesised images were labelled according to the House-Brackman scoring system by a human observer. This data formed the basis of our calculations on accuracy and error.

In order to counteract any interpersonal differences which may exist in the intensity of natural smiles in our test set, the extracted feature points were warped to the mean smile of the set using Generalized Procrustes Alignment (GPA) [8]. This technique aligns two shapes with respect to position, rotation and scale by minimising the weighted sum of the squared distances between the corresponding landmark points.

The results of our preliminary experimentation are shown in Figure 5 and Table 3. Figure 5 illustrates the results of our technique when compared to the hand labelled data; the $\mathrm{x}$-axis shows the hand labelled grades, while the horizontal bands illustrate the thresholds used by the decision phase of our algorithm. As a cross reference, color coded labels corresponding to the algorithm output are shown on the far right of the figure.

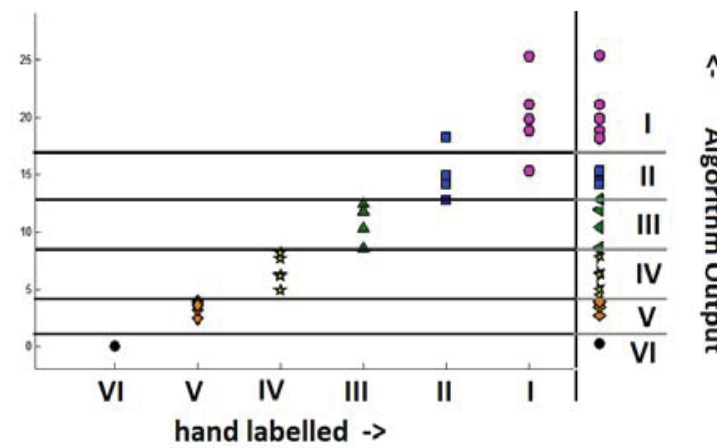

Figure 5: Illustrates the human labelled images (x-axis) when compare against the labels output by our system. The

horizontal lines represent the thresholds used in our algorithm for identifying the FMS damage. The output of the algorithm in terms of colour coded labels is displayed on the far right of the figure

Table 3 provides a breakdown of the $\%$ Accuracy and \% Error, showing an average accuracy of $87 \%$ and error of $13 \%$.

\begin{tabular}{|c|c|c|c|}
\hline Grade & Description & Accuracy & Error \\
\hline I & Normal & $88 \%$ & $12 \%$ \\
\hline II & Slight & $78 \%$ & $22 \%$ \\
\hline III & Moderate & $78 \%$ & $22 \%$ \\
\hline IV & $\begin{array}{l}\text { Moderately } \\
\text { Severe }\end{array}$ & $88 \%$ & $12 \%$ \\
\hline $\mathrm{V}$ & Severe & $90 \%$ & $10 \%$ \\
\hline VI & Total & $100 \%$ & $0 \%$ \\
\hline \multicolumn{2}{|c|}{ Average } & $87 \%$ & $13 \%$ \\
\hline
\end{tabular}

Table 3: \% accuracy and error of our automated labelling system when compared to the hand labels for each grade and also average results across the dataset

\section{Discussion}

Our preliminary test set contained neutral and smile expressions from 5 healthy subjects. For each subject appropriate levels of FMS damage was synthesised using AAMs. Prior to experimentation, the synthesised images were labelled according to the House-Brackman scoring system by a human observer. Results of these experiments show an average accuracy of $87 \%$ and error of $13 \%$ when compared with the hand labelled scores.

Even at this very early stage it is apparent that computer vision methods such as those implemented in this paper have the capability to provide a means for automatically scoring facial motor ability. While far more comprehensive studies are required to be emphatic in this assertion it is certainly looking very plausible that technology incorporating such artificial intelligence can help form the core for dedicated rehabilitation systems for use in the home and clinic. Our next step is to conduct the required studies before full incorporation into a prototype system which can be tested with end users.

It is interesting to note that the use of objective techniques for facial scoring and feedback such as those proposed here allow the subjective element of assessment to be removed from the process. Many researchers have commented and studied the inconsistencies which can arise due to the subjective nature of even an expert's judgement when it comes to scoring $[9,10]$. The removal of this subjectivity therefore can only be considered a positive and desirable goal, as illustrated in our preliminary experimentation computer vision techniques have the potential to achieve this.

It is worthy of comment also that a reported problem with current home training regimes is that they are primarily based on mirror feedback and patients who have experienced facial disfigurement find it distressing to spend long periods of time observing their face. Computer based systems offer a clear advantage over this type of technique as they can provide alternative sources of feedback which are less distressing to the individual.

\section{CONCLUSION}

The preliminary experiments described in this paper are best understood in terms of demonstration of a proof of concept. We feel that machine vision methods appropriately applied can have an impact on facial rehabilitation therapy. We have described how AAM models in particular appear to have promise in capturing facial gesture dynamics in such a way as to faithfully replicate a standard facial scoring method. It is clear that such a method may form the basis for a home rehabilitation system which may allow individuals to engage in therapy through a form of guided learning. We hope to develop this technology further as part of a complete facial rehabilitation system. 


\section{REFERENCES:}

[1] Neely JG, Neufeld PS. Defining functional limitation, disability, and societal limitations in patients with facial paresis: initial pilot questionnaire. AmJ Otol 1996;17:340-342.

[2] Vanswearingen J., Facial rehabilitation: a neuromuscular reeducation, patient-centered approach. Facial Plast Surg. 2008 May;24(2):250-9.

[3] Cootes T, Taylor CJ. Statistical models of appearance for computer vision. Technical report, Wolfson Image Analysis Unit, Imaging Science and Biomedical Engineering, University of Manchester, Manchester M13 9PT, October 2001.

[4] Shu He, Soraghan JJ, O'Reilly BF, Supervised Locally Linear Embedding (SLLE) for facial paralysis image sequence analysis. IEEE conference on Multimedia and Expo 2008, 49-52

[5] Miehlke A, Stennert E, Chilla R. New aspects in facial nerve surgery. Clin Plast Surg 1979;6:451-470

[6] de Ru, J. Alexander; Braunius, Weibel W.; van Benthem, Peter Paul G.; Busschers, Wim B.; Hordijk, Gerrit Jan, Facial Nerve Grading Facial Nerve Function: Why a New Grading System, the MoReSS, Should Be Proposed. Otology \& Neurotology, October 2006 - Volume 27 - Issue 7 pp 1030-1036

[7] House JW, Brackmann DE. Facial nerve grading system. Otolaryngol Head Neck Surg 93: 146-147.

[8] Gower JC, Generalised Procrustes Analysis. Psychometrika, 1975, 40:33-50

[9] Steven Burres, Ugo Fisch, The Comparison of Facial Grading Systems, Arch Otolaryngol Head Neck Surg. 1986;112(7):755-758.

[10] Susan E. Coulson, Glen R. Croxson, Roger D. Adams, Nicholas J. O'Dwyer, Reliability of the "Sydney," "Sunnybrook," and "House Brackmann" facial grading systems to assess voluntary movement and synkinesis after facial nerve paralysis, Volume 132, Issue 4, Pages 543-549 (April 2005)

\section{ACKNOWLEDGEMENTS:}

The authors would like to thank Dr. Charles Markham, Mr. John McDonald and Dr. John Ghent of the National University of Ireland Maynooth for their input and contribution to this paper. 